\title{
Participación ciudadana en la justicia penal. El valor documental y patrimonial de los juicios criminales juzgados por el Jurado en México D.F. (1869-1883)
}

Citizen Participation in Criminal Justice. The Documentary and Patrimonial Value of the Criminal Trials Judged by Jury in Mexico F. D. (1869-1883)

\author{
Andrés Alejandro Londoño Tamayo \\ Programa de becas posdoctorales \\ Instituto de Investigaciones Históricas \\ Universidad Nacional Autónoma de México \\ alejolondono32@gmail.com
}

Recibido: 9 de octubre de 2017 • Aprobado: 8 de noviembre de 2017

Cómo citar este artículo: Andrés Alejandro Londoño Tamayo, "Participación ciudadana en la justicia penal. El valor documental y patrimonial de los juicios criminales juzgados por el Jurado en México D.F. (1869-1883)", en Legajos. Boletín del Archivo General de la Nación, núm. 15 (enero-abril 2018), pp. 35-70.

\section{Resumen}

En este artículo se analizan facetas del modelo de participación ciudadana en la justicia promovido por el jurado popular en México D.F. y se presenta el avance de un proyecto de catálogo de expedientes criminales con jurado. En primer lugar, se realiza una exploración del discurso del jurado como derecho político y su debate historiográfico que se articula con un comentario del esquema procesal de la ley de jurados para delitos comunes sancionada en 1869. Y, en segundo lugar, se exponen los resultados del trabajo de catalogación de los expedientes criminales con jurado tramitados entre 1869 y 1883 del Fondo Tribunal Superior de Justicia del Distrito Federal del Archivo General de la Nación de México (AGN).

Palabras clave: jurado popular, delitos penales, participación ciudadana, México siglo XIX, juicio público y oral

\section{Abstract}

This article analyzes facets of the model of citizen participation in justice promoted by popular jury in Mexico F.D. and presents the progress of a catalog project of criminal records by jury. First, the article explores the discourse of the jury as a political right and its historiographic debate which is articulated with a commentary on the procedural outline of the law for juries for common crimes sanctioned in 1869. And, second, this article reveals the results of the effort in cataloguing the criminal records with juries processed between 1869 and 1883 by the Fondo Tribunal Superior de Justicia del Distrito Federal of the Archivo General de la Nación de México (AGN).

Keywords: Popular jury, criminal offenses, citizen participation, Mexico's 19th Century, public and oral trial 


\section{Introducción}

El establecimiento del jurado popular para delitos penales comunes en la ciudad de México D.F., en 1869, estuvo acompañado por la extensión de un discurso político que enfatizó su capacidad para promover la participación ciudadana. Sin la importancia adquirida por este discurso dentro la cultura jurídica -según indica el proceso previo de institucionalización del jurado-, no hubiese sido posible la fijación del primer modelo procesal mixto con jurado que operó en la Ciudad de México entre 1869 y 1929. El jurado, en este sentido, fue dispuesto tanto para generar mayores garantías a los incriminados como para dinamizar la participación ciudadana en la justicia y activar el gobierno local. Este segundo aspecto marcó un cambio importante respecto a los modelos de justicia previos, y a pesar de su importancia histórica-debido a las transformaciones que generó- ha sido escasamente estudiado por las ciencias humanas y sociales, y existe, también, un desconocimiento de sus fuentes de estudio. - El jurado ha sido estudiado especialmente como institución judicial y no como institución política.

Estos vacíos y la constatación del importante predominio político y judicial que tuvo la institución en la capital mexicana han impulsado la realización del presente artículo. En éste se realiza una aproximación al valor del jurado popular dentro del proyecto de construcción de la ciudadanía mexicana - partiendo de su reconocimiento como derecho político- y se expone un trabajo de catalogación de expedientes criminales con jurado, mismos que recuperan su valor como fuentes para el estudio de la participación en la institución.

En este texto se dará cuenta de la trayectoria que tuvo el ideario de la participación ciudadana en el jurado y de la complejidad de su fijación en México D.F. durante el siglo xix. El examen de esta trayectoria permitirá dimensionar la impronta que dejó la institución en la cultura jurídica y judicial de los capitalinos. Tras ello se examinará la discusión historiográfica actual en torno a la participación en el jurado, y se discutirá la importancia de realizar estudios que incluyan el análisis de los expedientes criminales. A partir del estudio 
de las fuentes y de la historiografía se ha establecido aquí, como hipótesis central, que la primera ley sancionada para México D.F., en 1869, configuró un modelo garantista y participativo, el cual tuvo una aplicación regular en los expedientes criminales tramitados en los juzgados penales mexicanos entre 1869 y 1883.

En este artículo también se ofrece un modelo de catalogación de los expedientes criminales con jurado que se encuentran depositados en el Fondo Tribunal Superior de Justicia del Distrito Federal (FTSJDF) del Archivo General de la Nación de México (AGN). ${ }^{1}$ El interés de trabajar dicho aspecto surgió a partir de constatar el desconocimiento de los expedientes correspondientes, en tanto género documental, con formas y contenidos específicos, y que no se encuentran ordenados. Los expedientes del FTSJDF identificados han logrado conservarse gracias al trabajo de archiveros y protectores del patrimonio mexicano, ${ }^{2}$ y en su conjunto destacan no solo por el volumen (cantidad de unidades) sino por la variedad (tipologías delictivas) y por el buen estado de conservación material de las piezas documentales. Tales aspectos y sobre todo el que "contengan" la primera y única experiencia de participación de la ciudadanía capitalina en la administración de justicia penal, les otorgan un valor histórico y patrimonial que amerita ser divulgado.

Considerando lo cual, el trabajo de catalogación que se expondrá en el tercer apartado contiene un formato de registro de datos centrado en la estructura del expediente criminal, y particularmente

\footnotetext{
${ }^{1}$ En adelante FTSJDF y AGN-México.
}

2 Agradezco el apoyo y la orientación brindadas en el AGN-México, por la doctora Yolia Tortolero Cervantes, directora del Archivo Histórico Central; por la licenciada Mariana Jiménez Muciño, jefa del Departamento de Organización y Descripción Documental, y por la licenciada Alina Argüelles González, jefa del Departamento de Control de Acervos Históricos y Registro Central. También agradezco el asesoramiento teórico y metodológico que para este trabajo fue realizado por las doctoras Elisa Speckman y Linda Arnold. Finalmente, aprecio especialmente la labor desempeñada por Omar Iván López Hernández quien viene realizando, en condición de auxiliar, un excelente trabajo de identificación, digitalización y catalogación. 
en la información que éste ofrece sobre la participación ciudadana. Es por ello que el catálogo en cuestión informará sobre quiénes fueron los ciudadanos que administraron justicia (registro de nombres), cómo participaron (registro de veredictos) y en qué medida esta participación fue respetada por las autoridades judiciales (registro de sentencia penal y revisión).

Este escrito realizará aportes sobre todo dentro del campo de estudios mexicanos relativos a la historia del derecho y de la justicia, y dentro de las áreas de la archivística y la documentación. En primer lugar, contribuirá a la recuperación de la impronta que dejó la institución y de los materiales ligados a su práctica que se encuentran olvidados a pesar de su valor. En segundo lugar, contribuirá a promover los estudios académicos sobre el jurado y a la proyección de estudios de catalogación y divulgación más amplios que cubran la larga temporalidad en la que su ejercicio estuvo en vigor en México (1869-1929). Y, en último lugar, aportará elementos que pueden ser de interés para el actual debate en torno a la práctica del sistema penal "acusatorio", vigente en la Ciudad de México desde el año 2015, en la medida en que ilustrará la manera como fue aplicado el primer modelo procesal "acusatorio" en la capital durante el siglo XIX.

La exposición se despliega en tres apartados. En el primero se aborda la configuración del discurso del derecho de participación en la justicia, a través de jurado, que tuvo lugar en México D.F., las perspectivas historiográficas actuales sobre la institución, al tiempo que se enfatiza el valor de los expedientes criminales con jurado para el estudio de la participación ciudadana en la administración de justicia. En el segundo se aborda el modelo procesal mixto con jurado diseñado en la Ley de 1869. Y, en el tercero, se presentan los resultados del proceso de catalogación de los expedientes con jurado ubicados en el FTsjDF. Para esto último se dará cuenta de aspectos como la cantidad, variedad y estado de los expedientes criminales; se indicará el diseño de catalogación definido y se ejemplificará la manera como se viene realizando el registro de datos en el catálogo. 


\section{El jurado en México D.F. en materia de delitos comunes durante el siglo XIX. Antecedentes, historiografía y perspectivas de estudio}

El juicio por jurado en la Ciudad de México para delitos penales comunes se estableció por primera vez en 1869. Tras ello la institución fue debatida y admirada por la protección de los incriminados y la participación de la ciudadanía que ella generaba; también se convirtió rápidamente en un símbolo del progreso político en las ideas democráticas que además daba estabilidad al gobierno. ${ }^{3}$ La institución del jurado dejó una impronta notable dentro del foro desde su establecimiento hasta su derogación, sesenta años después, en 1929; pero a pesar de ello terminó siendo olvidada y eludida durante el siglo xx. ¿ ¿Qué factores incidieron en su olvido institucional y social? Es un interrogante que tiene mucho sentido plantearse en la actualidad debido a que la institución fue excluida del modelo procesal mixto recientemente establecido en la Ciudad de México. Aun cuando la misma fue un referente histórico de éste. Es de indicar que la instauración de la institución del jurado en los estados latinoamericanos durante el siglo xix -México, Colombia, Perú, Chile, Bolivia, Venezuela- dio lugar a la fijación de los primeros modelos procesales acusatorios de carácter mixto en dichos marcos. En esta etapa de origen, sin embargo, no se hablaba de modelo acusatorio sino del jurado, y era este último el que integraba las características que en la actualidad conforman el denominado proceso penal acusatorio: oralidad del procedimiento,

${ }^{3}$ Sobre las transformaciones en la justicia durante el periodo del Porfiriato, véase Speckman, "Los jueces, el honor y la muerte", pp. 1411-1466.

${ }^{4}$ La supresión de la institución del jurado en México D.F. en 1929 según el estudio de Elisa Speckman, se basó en consideraciones como la de la preminencia de los jueces profesionales sobre los jurados (en la tarea de juzgar) y el de la sanción de fallos polémicos. Speckman, Del Tigre de Santa Fulia, la princesa italiana y otras historias, pp. 127-128. Un ejemplo de un fallo polémico fue el emitido en el juicio con jurado de María Teresa de Landa, en 1928. Véase Monroy, "Identidades perdidas: Miss México 1928”, pp. 151-156. 
sesión plenaria pública y oral, práctica de los principios de inmediación, contradicción y concentración. ${ }^{5}$ El estudio comparado entre jurado popular y una institución insigne del derecho mexicano como el Amparo, también puede ser importante para dimensionar el valor de las garantías dentro de la práctica procesal penal nacional. ${ }^{6}$

En la ciudad de México D.F. del último cuarto del siglo xix no se consideraba la supresión del jurado iniciada a partir de 1930, esto obedecía al valor atribuido al mecanismo para reemplazar el modelo procesal inquisitorial y por su vínculo con la participación ciudadana. También porque durante el siglo xix se desarrolló una cultura jurídica amplia en torno a la institución - como lo ha demostrado la historiografía del derecho y de la justicia ${ }^{-7}$ y ello resultaba decisorio para su vigencia dentro del orden jurídico. Para la extensión social del conocimiento y la práctica de la institución durante esta época resultó fundamental su defensa realizada por liberales-republicanos moderados y radicales, que se encargaron de generar proyectos de ley y de debatir públicamente sus principios y mecanismos.

En este apartado de contextualización realizaremos, en primer lugar, un abordaje panorámico en torno a los procesos de establecimiento del jurado en México D.F. en materia de delitos comunes, que permitirá dimensionar la relevancia de la institución en la discusión jurídica. Después expondremos algunas de las perspectivas historiográficas de estudio que han centrado el campo de la participación ciudadana. Y, como tercer aspecto, cerraremos este apartado con unos comentarios sobre el valor de los expedientes criminales con jurado

\footnotetext{
${ }^{5}$ Respecto al establecimiento del jurado popular en otros contextos latinoamericanos, véase Londoño, "El jurado popular en Colombia en el juicio criminal ordinario", pp. 204-212, y Londoño, "Juicios de imprenta en Colombia", pp. 75-85. Para una discusión sobre la trayectoria de los modelos procesales penales en México y sus características, véase González Villalobos, "Sistemas penales y reforma procesal penal en México", pp. 16-17; también a Hernández, "La reforma procesal penal federal en México", pp. 281-525.

${ }^{6}$ Lira, "El amparo colonial y el juicio de amparo mexicano", 1972.

${ }^{7}$ Speckman, "El jurado popular para delitos comunes", pp. 743-787.
} 
para generar nuevos conocimientos sobre la práctica de tal institución en los juzgados.

El jurado fue una institución debatida y practicada durante el siglo xix en materia de delitos comunes, como puede observarse a partir de fuentes como actas parlamentarias, literatura jurídica, hojas sueltas, expedientes criminales, etcétera. En ellas se plasmó el dinamismo generado por dicha metodología dentro del foro judicial y la actividad ciudadana en torno a su práctica. La promoción del jurado popular para "delitos comunes" en México D.F. tuvo dos momentos cruciales antes de su establecimiento en 1869, uno en la década de 1820 y otro en la de $1850,{ }^{8}$ en los que sectores de la elite liberal tramitaron notables proyectos de ley en el Congreso. En el primer momento, un conjunto de juristas entre los que destacaron José María Luis Mora, José María Jáuregui, Benito Guerra y Lorenzo Zavala, se encargaron de debatir los límites y posibilidades de establecimiento del jurado en México y de rechazar la argumentación conservadora contra el mecanismo. ${ }^{9}$ Estos juristas y políticos se nutrieron de un ideario del jurado desarrollado por los pensadores del derecho penal europeos, en el que se ponderaba su capacidad de ofrecer garantías procesales. ${ }^{10}$ De dicho ideario retomaron argumentos como el relativo a que el jurado daba más garantías que el procedimiento con juez letrado en tanto el "juicio" era

${ }^{8}$ Se ha explorado especialmente la discusión legislativa del Congreso de 1856-1857. Véase el estudio de González Oropeza, "El juicio por jurado en las constituciones de México", pp. 75-78.

${ }^{9}$ Véanse los discursos en torno al jurado sostenidos por José María Luis Mora, José María Jáuregui y Benito Guerra en el Congreso Constituyente de 1826. México, Actas del Congreso Constituyente del Estado libre de México (t. VIII, pp. 31, 372, 755-780, t. XIX, pp. 19-30.). Véanse escritos de Mora, "Obras Sueltas", p. IV, y Zavala, Viaje a los Estados Unidos de Norte América, pp. 151-152, 246.

${ }^{10}$ Entre éstos se encuentran los autores de la escuela clásica del derecho penal: Beccaria, Tratado de los delitos y las penas, p. 51; Filangieri, Ciencia de la legislación, t. IV, p. 283. Jeremy Bentham, a diferencia de los autores anteriores, no propuso el establecimiento del jurado: Bentham, Tratados de legislación ciwil y penal, vol. 4, pp. 356-357. Sobre la presencia de las ideas de Bentham dentro del liberalismo mexicano, véase Hale, El liberalismo mexicano, pp. 152-193. 
hecho por un grupo y no por un solo sujeto, y porque los jurados eran elegidos al azar y no nombrados por el Poder Ejecutivo. ${ }^{11}$ También retomaron el argumento relativo a la perfección del procedimiento criminal con jurado en tanto integraba garantías como el sorteo y la recusación, el doble panel de jurado (acusación y calificación), y un veredicto fundado en el criterio de unanimidad. Y, por último, en las obras de los publicistas europeos los liberales mexicanos también pudieron observar que el jurado era valorado por la posibilidad que generaba de anular la ley cuando se trataba de conseguir una administración de justicia más equitativa y acorde con las circunstancias particulares de los sujetos. ${ }^{12}$ Este ideario del jurado como institución garantista - a pesar su amplia extensión y aceptación dentro de la elite liberal mexicana- no fue suficiente para que ésta concretara el establecimiento del mecanismo para el juicio de los delitos comunes. Al igual que la lógica reflejada durante la discusión del proyecto de Ley de Jurados para Delitos Comunes, en el Congreso de 1826, la introducción del mecanismo no fue posible, sobre todo porque se consideraba que se requería la sanción y afianzamiento de otros medios como el Código Penal y el Procesal, y la organización de los juzgados. ${ }^{13}$

Un segundo momento de promoción del jurado para el juicio de los delitos comunes tuvo desarrollo en el Congreso de 1856-1857, y como en el caso anterior las acciones realizadas no terminaron decidiendo la fijación del mecanismo. En este marco un conjunto de liberales como Francisco Zarco o José María Mata sostuvieron que el establecimiento de la institución no solo era importante por el elemento garantista que ofrecía - como se había indicado en 1820-, sino por su aporte a la dinamización de la política local. ${ }^{14}$ Desde su perspectiva, la institución contribuía

11 Este argumento se desarrolló después de las transformaciones realizadas por Napoleón al modelo de jurado francés fijado a partir de 1791. Véase Comte, De las Facultades y Obligaciones de los furados, pp. XII-CXXXVI (discurso preliminar).

${ }^{12}$ Constant, Curso de política Constitucional, pp. 290-300. Véanse las referencias sobre la influencia de Constant en Hale, El liberalismo mexicano, pp. 58-73.

${ }^{13}$ México, Actas del Congreso Constituyente del Estado libre de México, t. xix, pp. 19-30.

${ }^{14}$ Zarco, Historia del Congreso Constituyente de 1856 y 1857, t. 2, p. 293. 
a un proceso de transformación institucional y social en la medida en que generaba el "derecho político" de participación en la justicia, y en tanto atribuía a la ciudadanía la administración de justicia. Estos liberales compartieron con otros juristas del periodo una comprensión del jurado como un derecho político cuya participación debía regularse de manera similar a la del voto, y cuya fijación derivaba ineludiblemente en el aumento del poder ciudadano sobre el gobierno. La posibilidad de practicar una justicia autónoma, participativa y garantista que se concretaba mediante jurado -según aquellos liberales- era suficiente para introducir la institución en el orden jurídico. ${ }^{15}$ Pero, a pesar de la promoción que realizaron para su establecimiento en materia de delitos comunes en la Constitución de 1857, la iniciativa fue derogada. $\mathrm{Su}$ rechazo dentro del Congreso se basó en el argumento relativo a que la institución daba lugar a una aplicación legal heterogénea que contradecía la emitida desde el Poder Legislativo (positiva, homogénea, general), lo que era inevitable por la diversidad de la población que participaba en el jurado -según fue argumentado por liberales como Isidoro Olvera o Ignacio L. Vallarta- Éstos sostuvieron, además, que la sociedad mexicana no se encontraba preparada para la introducción del jurado. ${ }^{16}$

La institucionalización del jurado para la Ciudad de México se concretó finalmente en el año 1869 tras la caída de la monarquía. En este marco de tránsito el naciente gobierno defendió el jurado como uno de los símbolos del federalismo y la democracia. En cuanto a la primera Ley de Jurados en Materia de Delitos Comunes para la Ciudad de México de 1869, consideramos que marcó un hito dentro de la historia del derecho mexicano debido a que trasformó radicalmente la práctica del proceso penal. Esta transformación se vio reflejada en la ampliación de las garantías procesales a los incriminados, en el

${ }^{15}$ En el Congreso Constitucional de 1856 y 1857 los promotores del Jurado defendieron su establecimiento, entre otras cosas porque consideraron que ampliaba la participación ciudadana. Se planteó, incluso, que los sectores indígenas podían tener el derecho de jurado como fue la opinión de Guillermo Langlois y José María Mata. En Zarco, Historia del Congreso Constituyente de 1856 y 1857, t. II, p. 515.

${ }^{16}$ Zarco, Historia del Congreso Constituyente de 1856 y 1857, t. II, pp. 167-180. 
canal que abrió para la participación ciudadana en la administración de justicia y en la atribución que hizo a ésta de la jurisdicción de la mayoría de los delitos penales comunes.

Teniendo presente la anterior contextualización sobre el establecimiento del jurado en México D.F. - que advierte la importancia adquirida por el mecanismo dentro de la cultura jurídica y judicial- pasamos a realizar una referencia de algunos estudios historiográficos actuales que han debatido sobre la trayectoria del mecanismo. Los estudios sobre el jurado se publicaron, sobre todo, durante el periodo en que estuvo en vigor en el orden jurídico - producidos especialmente desde la ciencia jurídica ${ }^{-17}$ y dejaron de ser realizados de manera notable tras su derogación en 1929. Estos estudios resurgieron durante la década de 1990, siendo alentados en buena medida por el debate que promovió el proyecto de sustitución del modelo procesal inquisitorial por un modelo acusatorio. La producción académica generada en este marco, sin embargo, no resultó relevante para la inclusión del jurado en el modelo procesal inquisitorial que se estableció. ${ }^{18}$

Dentro de los estudios historiográficos contemporáneos encontramos los de corte jurídico en los que se analiza la regulación de los modelos de jurado establecidos durante el siglo xix y sus procesos de establecimiento. ${ }^{19}$

${ }^{17}$ Un balance acerca de los estudios sobre el jurado en Speckman, "El jurado popular para delitos comunes", pp. 743-787. Una de las aportaciones más notables desde la ciencia jurídica sobre el jurado en México la realizó Demetrio Sodi, véase su análisis de los argumentos en pro y en contra del jurado popular y el relativo al Artículo 14 constitucional (Los hechos declarados por el jurado, son irrevocables) Sodi, El jurado popular en México, pp. 393-427.

${ }^{18}$ En México la sustitución del modelo inquisitorial por un modelo acusatorio de carácter mixto se realizó paulatinamente desde el año 2006. En la actualidad este último se encuentra en vigor en la mayoría de los estados de la Federación. Estados Unidos Mexicanos, Código Nacional de Procedimientos Penales. Última Reforma en el DOF 17-06-2016 (Arts. $1^{\circ}-4^{\circ}$ ).

${ }^{19}$ Ovalle Favela, "Los antecedentes del jurado popular en México", pp. 747-786; Arroyo Padilla, "Los jurados populares en la administración de la justicia en México en el siglo XIX". Nueva época 47 (2000), pp. 137-169; Rabasa, "El jurado popular en las constituciones de 1857 y 1917”, pp. 555-577; González Oropeza, "El juicio por jurado en las constituciones de México", pp. 73-86; Speckman, "El jurado popular para delitos comunes”, pp. 743-787. 
También se hallan trabajos desde la historia crítica del derecho que indagan la exclusión del jurado en los inicios del Estado constitucional, por parte de las elites liberales hispanoamericanas. Autores como Bartolomé Clavero han indicado que la exclusión del jurado del orden jurídico significó que el Poder Legislativo se convirtiera en un poder superior, en la medida en que no tenía un contrapeso en el Poder Judicial cuando se trataba de la aplicación de la ley. Este contrapeso sí existía según dicho autor en los Estados constitucionales inglés y estadounidense donde el jurado era un mecanismo que lo efectuaba; sin embargo, ello no fue atendido por los liberales de países europeos como España o Francia o de los países hispanoamericanos. ${ }^{20}$ Bartolomé Clavero también ha planteado la dificultad que implicaba la práctica del jurado en México en los orígenes del Estado, debido a cuestiones como la "diversidad" de la población: las diferencias culturales de los jurados integraban una "heterogeneidad" dentro del ejercicio de administrar justicia que chocaba con una codificación republicana tendente a la "homogeneidad" legal.

Por último, destacamos la perspectiva de la historia crítica del derecho y la justicia que ha reconstruido el impacto que la institución tuvo dentro de la cultura jurídica mexicana (no sólo la del foro), y ha demostrado que el mecanismo dio lugar a una pluralidad jurídica y judicial en tanto justicia de particulares (ciudadana). ${ }^{21}$ Los trabajos de Elisa Speckman indican la centralidad que tuvo la institución en los inicios del Porfiriato (1876-1911) en la Ciudad de México y esclarecen su incidencia en el proceso de modernización de la justicia.

Perspectivas como las anteriores han arrojado importantes luces sobre el valor histórico del jurado en México en razón de los procesos de transformación jurídica y judicial que produjo. Pero a pesar de

\footnotetext{
${ }^{20}$ Clavero, Happy Constitution, pp. 250-250.

${ }^{21}$ Speckman, "Los jueces, el honor y la muerte", pp. 1411-1466. Véanse las referencias en torno al Jurado y la práctica de la administración de justicia de México D.F. en Piccato, Ciudad de sospechosos, pp. 127-164. Véase un interesante estudio de caso en el que se aborda el funcionamiento de la institución en el año de su derogación en Rojas Sosa, "El caso de la 'fiera humana", pp. 234-238.
} 
los aportes, no se ha profundizado lo suficiente sobre las prácticas y dinámicas generadas por la institución en materia de participación ciudadana. Estudiar este aspecto aportaría a una mejor comprensión sobre la manera como los contemporáneos aplicaron la institución, y sobre los límites y posibilidades de su práctica en el marco del Poder Judicial y de la sociedad mexicana. Consideramos que su abordaje es importante para matizar tópicos sobre el procedimiento que señalan que fue aplicado erróneamente o que no tuvo posibilidad debido a la diversidad de la población, para cuya matización se vienen realizando aportes historiográficos significativos. ${ }^{22}$

El estudio sobre la práctica de la institución del jurado en México exige, no obstante, un trabajo previo de visibilización y ordenamiento de fuentes documentales que son idóneas para dar cuenta de dicho aspecto, que no han sido estudiadas por la historiografía - exceptuando la perspectiva de la historia del derecho y de la justicia-. Entre éstas, las más importantes son, por un lado, las que fueron producidas por el ayuntamiento durante su gestión administrativa del derecho político de participación en el jurado: censo de jurados, registros de datos de domicilio de los ciudadanos (padrón), listas de exclusión del cargo, comunicaciones con el juzgado $;^{23} \mathrm{y}$, por otro lado, las que fueron producidas por el juzgado letrado en su tarea de aplicación del mecanismo: libros de programación de juicios, listas de jurados y expedientes criminales.

${ }^{22}$ Los trabajos de Marta Irurozqui ilustran sobre el dinamismo ciudadano que acompañó la práctica política en el marco de construcción de los Estados republicanos hispanoamericanos, al igual que precisan la amplitud y complejidad de la participación en prácticas como la del voto. Su reconstrucción historiográfica de categorías como la de ciudadano son indicativas de un sujeto jurídico activo en el ámbito electoral, en la justicia y en la guerra. Véase Irurozqui, "El espejismo de la exclusión”, pp. 57-92; Irurozqui, La ciudadanía en debate en América Latina, pp. 14-77; e Irurozqui, Sangre de ley, pp. 7-24. Sobre el dinamismo jurídico-político en México y su importancia para la participación en la justicia véase Marino, Huixquilucan ley y justicia en la modernización del espacio rural mexiquense, 1856-1910, pp. 225-243.

${ }^{23}$ Los documentos producidos por el Cabildo de la Ciudad de México en relación con el jurado se encuentran catalogados en Arnold, Catálogo electrónico preliminar del Archivo Histórico, 2000. 
Estos últimos, en particular, resultan esenciales para entender las dinámicas y regularidades de la práctica de la institución porque contienen la acción realizada tanto en el procedimiento de instalación del panel de jurado como en el desarrollo del juicio oral. Se trata de documentos que dan cuenta de quién participó efectivamente en el jurado, cómo lo hicieron y la intervención de las autoridades; que permiten observar si se aplicaron las garantías procesales de la institución, y que indican los procedimientos seguidos durante el juicio oral. El valor histórico y patrimonial de estas fuentes exige, por tanto, la realización de investigaciones que aporten a su conocimiento, conservación y difusión.

\section{El diseño garantista y participativo de la Ley de Jurados de 1869 y su expresión en los expedientes criminales tramitados en los juzgados penales de la México D.F. (1869-1883)}

La Ley de Jurados sancionada para la Ciudad de México en 1869 cristalizó un ideal perseguido desde el inicio de la República. Esta ley constituye un hito para la historia del derecho mexicano no tanto por ser la primera regulación orgánica del jurado para delitos comunes de la Ciudad de México, sino porque en ella se atribuyó una amplia competencia al jurado sobre los delitos y se fijaron nuevos esquemas procesales encaminados a transformar la práctica de la administración de la justicia penal que había imperado durante el Periodo Colonial y el Republicano. ${ }^{24}$ Esta ley que implicó el reemplazo del modelo inquisitorial por uno de carácter acusatorio se mantuvo con apenas leves alteraciones hasta la Ley Orgánica del Jurado sancionada en el año 1891. ${ }^{25}$ La Ley de Jurados de 1869 otorgó, por primera vez en México, una competencia a la ciudadanía para juzgar sobre el "hecho" en la

${ }^{24}$ Ciudad de México, Ley de Furados en Materia Criminal para el Distrito Federal, pp. 1-33.

${ }^{25}$ En el Código de Procedimientos Criminales de 1880, se modificó el proceso de elección de los jurados con la finalidad de hacerlo más garantista para los incriminados. México, "Código de Procedimientos Penales del Distrito Federal", pp. 346-371. 
mayoría de los delitos penales graves: robo, hurto, homicidio, violación, estafa, incendio, incesto, lesiones, plagio, rapto, parricidio, etcétera. Mediante ello se sustrajo una competencia antes atribuida a los jueces letrados y se otorgó un poder a la ciudadanía dentro de la justicia, que no había tenido lugar en la historia de la Ciudad de México. A pesar de ello, la introducción del jurado no planteaba una exclusión del juez, sin cuya participación la institución no podía ser operativa; lo que perseguía era la aplicación del principio garantista de la división judicial tan debatida durante el periodo. ${ }^{26}$ En la práctica de la institución "el derecho" debía ser indicado por los jueces a quienes correspondía definir la tipificación delictiva a juzgar por los paneles -en el caso de México la fuente la constituyó el Código Penal de $1871-{ }^{27}$ y realizar la sentencia penal con la respectiva graduación de la pena.

En cuanto al modelo procesal, la Ley de Jurados de 1869 sancionó un esquema mixto con mayor énfasis acusatorio ordenado en dos etapas: instrucción sumarial y juicio oral con jurado. En la etapa de instrucción las autoridades debían realizar toda la actuación probatoria, y, en el juicio oral correspondía a los jurados la emisión de un veredicto a partir de los materiales probatorios expuestos en el juicio y fundado en conciencia. Pasamos a indicar los trámites prescritos por dicha ley para cada una de estas etapas, cuyas diligencias debían registrarse en los expedientes criminales.

En el modelo procesal mixto con jurado se prescribió, primero, una instrucción sumarial cuya realización se encargó al juez y al funcionario del Ministerio Público. En ésta se registraban las acciones de investigación encaminadas a probar el delito y su autor: interrogatorios, peritajes, requisitorias, emplazamientos, etcétera. La Ley de Jurados trasladó las ratificaciones y careos de testigos de la fase

${ }^{26}$ González, "el juicio por jurado”, p. 106. La idea de división judicial consistía en atribuir a diferentes personas la función de hallar el crimen, la de presidir el juicio y la de pronunciar y exponer la ley. Se discutía que cuando estas acciones eran realizadas por una misma persona no podía existir garantía procesal.

${ }^{27}$ México, Código penal para el Distrito Federal y Territorio de la Baja California, pp. 112-300. 
de instrucción sumarial en la que tenían un registro escrito - como se realizaba en el modelo inquisitorial previo- a la sesión de juicio con jurado en la que pasaron a tener una dinámica pública y oral. La sumaria se iniciaba por jueces de primera instancia de pueblos como San Ángel, Xochimilco, Coyoacán, Tlalpan o por el juez penal letrado de la capital; sin embargo, si la realizaban los primeros, debían remitirla al Juzgado Penal de México debido a que solamente el juez letrado, apoyándose en el Ministerio Público, determinaba el mérito para el juicio oral o el sobreseimiento. La sumaria no fue prescrita como el fundamento exclusivo que debía seguir el jurado para la sanción del veredicto. Su valor como mecanismo que fundamentaba el paso al juicio oral radicaba en que servía al juez para definir la tipificación delictiva y para instruir la sentencia penal. Para el jurado, por su parte, la sumaria era un medio informativo para la resolución del veredicto. Ésta debía leerse en el inicio del juicio, acto establecido para extraer la información que aportaba a la resolución de la causa; no obstante, la sumaria no era el único medio para la interpretación, ya que el panel también tenía que tener en cuenta el debate oral. ${ }^{28}$

Terminada la etapa de instrucción sumarial debía proseguir la segunda etapa, consistente en el juicio público y oral con jurado; la cual requería, no obstante, que los jueces contaran con las listas de jurados para realizar el sorteo del panel titular del juicio. La Ley de 1869 estableció un orden estricto en la preparación de dichas listas cuya finalidad era hacer garante el proceso de participación ciudadana que se generaba mediante el jurado. Debe recordarse que para este momento histórico la institución del jurado se concebía también como un derecho político similar al voto. Pasamos a dar cuenta de la manera como la Ley de Jurados de 1869 ordenó el procedimiento de elección de los ciudadanos que fue encargada al cabildo y el de formación de

${ }^{28}$ México, Ley de Jurados, p. 6. Art. 15: "El día de la vista, que será pública, se constituirá el jurado bajo la presidencia del juez de lo criminal, y se dará lectura al sumario estando presentes las partes y todos los testigos". 
los paneles que se atribuyó a los jueces, para luego pasar a precisar el ordenamiento que se hizo del juicio público y oral.

La mencionada ley atribuyó al cabildo la tarea de realizar el censo de ciudadanos aptos para la participación en el jurado y de confeccionar la lista de elegidos por año, tareas que le demandaron al ayuntamiento la realización de actividades como el empadronamiento de ciudadanos (se requería un dato preciso de nombre y apellido y dirección de residencia), la publicidad de los elegidos y la atención de las solicitudes de excepción del encargo. Esta ley estableció que cada año, durante el mes de diciembre, el ayuntamiento debía "insacular" o fijar una lista de seiscientos ciudadanos con los requisitos legales para ser jurados. Esta lista según el Artículo 66 debía dividirse en cuatro listas de 150 jurados, que posteriormente se sorteaban para determinar su servicio durante los trimestres del año. El ayuntamiento fue el encargado de dicha tarea debido a que se concebía como una institución representativa de la sociedad, electa por los ciudadanos, y no designada por el Poder Ejecutivo local.

Para ser jurado dicha ley estableció como requisitos ser ciudadano mexicano, vecino de la capital, mayor de veinticinco años, no ser tahúr o ebrio consuetudinario ni tener antecedentes por delitos penales comunes. Se prohibió la elección de médicos, funcionarios públicos y de quienes no pudieran disponer de su tiempo sin privarse de jornal. Fueron excluidas las mujeres. Los requisitos de participación fijados en la citada ley fueron flexibles respecto a los establecidos para el modelo de jurado de imprenta en vigor durante la primera mitad del siglo xix, sin embargo, no lo fueron tanto como para abrir la participación a mayorías sociales. ${ }^{29}$ Coincidimos con este planteamiento en tanto que, para un sector social como el indígena, aunque no se estableció legalmente su exclusión, la ley expuesta, por sí misma no modificaba las condiciones sociopolíticas que la imponían en la práctica.

${ }^{29}$ Speckman, Del Tigre de Santa Fulia, La princesa italiana y otras historias, pp. 103-110. 
Por su parte, en cuanto a la formación de los paneles para presidir los juicios, la expresada ley fijó dos actuaciones consecutivas, en primer lugar, ordenó a los jueces letrados entregar al incriminado y al fiscal la lista trimestral que contenía 150 jurados para que efectuaran su "derecho" a recusar: podían eliminar de ésta hasta 12 jurados sin presentar motivación. Y, en segundo lugar, encargó a los jueces la realización del "sorteo" de los 13 jurados que serían llamados para el juicio: 11 titulares y dos suplentes.

En la segunda etapa, relativa al juicio público y oral la nombrada ley estableció los procedimientos de lectura del expediente, declaración del incriminado, interrogatorio de testigos y debate entre fiscal y defensor. Su función radicaba en instruir a los jurados para la emisión del veredicto sobre el hecho, que debían emitir tras una deliberación en privado. Para la sanción el juez preparaba un cuestionario que, conforme con el modelo fijado por esta ley debía contener, en primer lugar, una pregunta relativa a la autoría del imputado sobre el hecho criminal, y, en segundo lugar, un conjunto de preguntas sobre las circunstancias atenuantes y agravantes de la comisión del delito. Además, indicó que el desarrollo de este cuestionario era en acto deliberativo, secreto, indivisible en tanto limitado temporalmente; y prescribió la unanimidad de seis votos de los jurados de entre los 11 para la resolución positiva o negativa de cada una de las preguntas (véanse imágenes 4 y 5 sobre el veredicto). La resolución del cuestionario no debía presentar contradicciones notorias que dificultaran al juez la sanción de la sentencia, por lo que se le otorgó al juez la facultad de interrogar en una segunda ocasión al jurado para esclarecer contradicciones. El juez no podía intervenir, en cambio, cuando se producía la existencia de una contradicción entre la respuesta del jurado sobre el hecho y el desarrollo de la sumaria. Por ejemplo, en los casos en que resultaba terminantemente probado en la sumaria la comisión del delito y se concluyera lo mismo en el juicio oral y el jurado indicaba en la primera pregunta del cuestionario que el incriminado no era culpable. Finalmente, en el término de 24 horas el juez debía sancionar la sentencia penal, para luego 
remitir el expediente a la revisión por parte del Tribunal Superior del Distrito. ${ }^{30}$

La Ley de Jurados en Materia Criminal para el Distrito Federal dio lugar a una práctica procesal compleja que exigió la cooperación entre ciudadanos, autoridades judiciales y ejecutivas del gobierno local. Los procedimientos establecidos fueron plasmados por las autoridades en diferentes formatos documentales que diseñaron en aras de agilizar y lograr un mejor registro de los trámites. Estos modelos documentales no fueron prescritos por la ley antes referida, sino que surgieron durante el transcurso de la práctica hasta lograr institucionalizarse y, en algunos casos, pasaron a ser formatos impresos.

En definitiva, la Ley de jurados en materia criminal para el Distrito Federal estableció un modelo procesal mixto que priorizó el elemento "acusatorio" tal como lo señala el valor otorgado al juicio con jurado. El mecanismo se aplicaba en el marco de un juicio oral diseñado para la controversia y la sanción del veredicto del jurado sobre el hecho; el panel se reunía para deliberar en privado (habitación cerrada) y responder el cuestionario, a lo que seguía la sanción del juez letrado sobre el derecho. Estas labores de carácter deliberativo y oral modificaron la administración de justicia en materia penal si se

${ }^{30}$ Flores, "Sobre la fundamentación de las sentencias y el arbitrio judicial", pp. 226229. Véanse los cuadros 6 y 7 realizados por la autora en los que se indican las leyes utilizadas por los jueces para fundamentar sus sentencias entre 1869 y 1872. En ellos se constata que los jueces criminales de la capital fundaron sus sentencias en la ley de jurados de 1869. En la práctica hubo dos formas recurrentes de aplicación de esta ley por parte de los jueces, por un lado, cuando los veredictos fueron absolutorios, sancionaron la resolución del expediente según la Ley del 15 de junio de 1869; y, por otro lado, cuando los veredictos fueron condenatorios, indicaron además de esta ley la legislación penal regulatoria de la pena -la Ley del 5 de enero de 1857 y el Código Penal de 1871-. Las tesis de la historiografía del derecho sobre el alcance del ideario del positivismo jurídico y la dinámica del arbitrio judicial, resultan relevantes para dimensionar la complejidad de la organización de la justicia y el impacto que dentro de ésta tuvo el establecimiento del jurado. Véase Garriga y Lorente, Cádiz, 1812, La constitución jurisdiccional, pp. 373-421; Garriga, Historia y Constitución, pp. 59-106; y López, La organización para la administración de la justicia ordinaria en el Segundo Imperio, 2014. 
consideran las características del modelo procesal-penal inquisitorial previo: escrito, secreto, sin división de acción judicial (el juez instruye, establece el hecho y el derecho), con un sistema probatorio basado en reglas y medidas (prueba semiplena/prueba plena).

La práctica procesal reflejada en los expedientes criminales estudiados para este artículo indica que el veredicto de los jurados sobre el hecho criminal fue terminante para la resolución condenatoria o absolutoria de las causas. Los jueces no modificaron este dictamen ni tampoco el Tribunal Superior (instancia revisora de todas las causas) que a su vez se mostró respetuoso con el mismo. Esta práctica indica, en definitiva, una asunción del veredicto del jurado como una expresión de la soberanía popular que debía ser respetada y acatada. Nos interesa señalar este aspecto porque refleja el dinamismo jurídico promovido por el jurado y su efecto para una justicia plural.

\section{Los expedientes criminales del Fondo Tribunal Superior de Justicia del Distrito Federal del Archivo General de la Nación de México. Balance y perspectivas de catalogación $(\mathbf{1 8 6 9 - 1 8 8 3})$}

Los expedientes criminales con jurado ostentan un importante valor histórico y patrimonial debido a que contienen la práctica de un modelo de administración de justicia penal caracterizado por la "participación ciudadana" y la aplicación de garantías procesales. Se trata de una fuente que aporta a la comprensión histórica de la dinámica del proceso penal mixto con jurado en la capital mexicana, y acerca de los límites y posibilidades de su práctica. A pesar de este importante valor, dichos expedientes son escasamente conocidos, como es el caso de los que se hallan en el FTSJDF del AGN-México.

Los expedientes con jurado de dicho fondo vienen siendo el tema central de una investigación que ha tenido como objetivo su catalogación y divulgación, con el propósito de facilitar su acceso. Ésta lleva dos años de desarrollo con labores de identificación, sistematización, digitalización y catalogación. Aquí se presentarán algunos de los resultados obtenidos: por un lado, sobre aspectos como la cantidad, 
variedad y estado material de los expedientes; y, por otro lado, una explicación sobre el proceso de catalogación. Finalmente, se mostrará un ejemplo del formato de catálogo que se viene realizando.

Los expedientes que hasta la fecha se han identificado y digitalizado son 713, entre los años 1869 y 1883. La identificación se hizo durante la revisión de 410 cajas en las que estaban contenidos 119,795 documentos de diferente tipo elaborados por los juzgados letrados de la capital (juicios civiles, criminales, comerciales y documentos de orden del juzgado). Las revisiones del FTSJDF posteriores a 1883 indican que contiene expedientes criminales con jurado hasta la derogación del mecanismo en 1929. El volumen de expedientes identificados es apenas una pequeña parte de los expedientes con jurado tramitados por los juzgados criminales de la capital mexicana, porque según los libros de programación de los juzgados, el número de expedientes de este tipo fue muy superior. Esta gran cuantía no es de extrañar si se tiene en cuenta que la institución se estableció como mecanismo exclusivo de juicio para los delitos penales comunes, que eran frecuentemente demandados ante las autoridades.

En cuanto a la variedad, se indica que dentro del grupo de 713 expedientes identificados existe una diversidad que se corresponde con las tipologías criminales penales juzgadas por el jurado. Según la tipología criminal (robo, homicidio, heridas) hay una diferencia de trámite tanto en la investigación sumaria como en el juicio oral (son diversos los métodos de prueba y defensa utilizados por autoridades y defensores). Los trámites plasmados en los expedientes son importantes por la multiplicidad de información que ofrecen sobre aspectos del ámbito criminal y de la administración de justicia, y por lo que pueden reflejar sobre el funcionamiento de los juicios orales.

Como se observa en la Tabla 1 el mayor número de expedientes criminales hallados durante los años que enmarcan nuestra investigación fueron los relativos a los delitos de lesiones, robo y homicidio. Esto indica que la institución del jurado administró justicia sobre todo en los delitos penales más graves tipificados en el Código Penal que entró en vigor en 1872. En segundo lugar, se halló una cantidad importante de expedientes con tipologías vinculadas con la agresión 
sexual como las de estupro, violación y rapto. Y finalmente, se encontró una cifra reducida de expedientes criminales con jurado para tipologías criminales leves como: amagos, abuso de confianza, agresión a la policía, calumnia, allanamiento de morada.

Tabla 1. Tipificaciones delictivas de los expedientes con jurado tramitados entre 1869 y 1883 depositados el Fondo Tribunal Superior de Justicia del Distrito Federal del AGN-México

\begin{tabular}{|c|c|}
\hline Aborto & 1 \\
\hline Abuso de autoridad & 1 \\
\hline Abuso de cofianza & 5 \\
\hline Agresión a la policía & 7 \\
\hline Allanamiento de morada & 3 \\
\hline Amagos & 1 \\
\hline Calumnia & 1 \\
\hline Estafa & 1 \\
\hline Estupro & 7 \\
\hline Homicidio & 186 \\
\hline Hurto & 1 \\
\hline Incendio & 1 \\
\hline Incesto & 1 \\
\hline Infanticidio & 5 \\
\hline Lesiones (heridas, riñas, golpes) & 378 \\
\hline Parricidio & 1 \\
\hline Peculado & 1 \\
\hline Plagio & 4 \\
\hline Plagio y hurto & 1 \\
\hline Rapto & 1 \\
\hline Rapto y estupro & 3 \\
\hline Robo & 79 \\
\hline Violación & 22 \\
\hline Violación de correspondencia & 2 \\
\hline Total de expedientes & 713 \\
\hline
\end{tabular}


En cuanto al estado material, los expedientes señalan buen estado de conservación y enseñan un proceso de modernización de los formatos diseñados para los autos judiciales. Casi la totalidad de los expedientes hallados se encuentran completos de inicio a fin, desde la carátula y los mandatos judiciales iniciales de sumario hasta el auto de revisión sancionado por el Tribunal Superior del Distrito Federal. La mayoría se hallan libres de hongos, mutilaciones o manchas. Estos expedientes también destacan por tener una caligrafía legible y una integración precisa de estos autos, lo cual facilita la lectura y la identificación de la información. Respecto al ejercicio de catalogación, presentaremos, primero, el diseño de formato de catálogo, dando cuenta de las variables de descripción fijadas, y luego, se ofrecerá un ejemplo de la catalogación que se viene realizando. Se enfocaron tres categorías para la descripción documental del expediente, en primer lugar, la ubicación y estado material: número (núm.) de caja y núm. de expediente, estado material, cantidad de folios y descripción de contenido. En segundo lugar, datos del proceso criminal: fechas extremas, lugar del hecho, víctima/victimario, nombres de funcionarios judiciales (juez, fiscal, abogado). Y, en tercer lugar, datos relativos a la participación ciudadana: nombres de jurados, veredicto sancionado, sentencia y revisión de corte. Estos últimos datos constituyen el elemento de mayor importancia de este catálogo en la medida en que pretenden indicar cómo se desarrolló la participación ciudadana. Realizamos una explicación sobre cada uno de los datos referenciados en el catálogo.

1. Núm. de caja. Este dato refiere la caja de la que fue extraído el expediente criminal. Debe tenerse en cuenta que los expedientes se encuentran depositados en cajas que siguen un orden numérico ascendente que parte del año 1821, y que éstos no están ordenados dentro de las cajas.

2. Núm. de expediente. Este dato referencia el número asignado por el juzgado penal letrado al expediente; el cual se ubica regularmente en el centro de la carátula de dicho documento.

3. Cronología. Se alude al inicio y al fin del expediente. La fecha de inicio se extrajo del auto de detención de policía o de la 
primera declaración del denunciante o fiscal del caso; la de cierre, del auto de sentencia del juez penal letrado.

4. Delito. Informa el delito o delitos juzgados; que es extraído de la carátula del expediente o del auto de cuestionario realizado por los jueces.

5. Lugar. Alude a la localidad en la que ocurrió el delito. Su indicación es importante debido a que los delitos sucedieron en la Ciudad de México y en pueblos aledaños como San Ángel, Coyoacán, Tlalpan, etcétera.

6. Demandante. Refiere al sujeto que interpuso el denuncio, que no siempre se correspondió con la víctima o el ofendido (los denunciantes también pueden ser testigos o familiares).

7. Demandado. Refiere el nombre del agresor o agresores, que no siempre se corresponde al indicado en la carátula del expediente. Esto hizo necesario cotejar el nombre del sujeto criminal indicado en la carátula con el denunciado en el auto de juicio oral.

8. Juez. Hace mención del nombre del juez penal letrado de la capital a quien se asignó el expediente. No se indicó el nombre del alcalde del pueblo o municipio cuando fungieron como jueces instructores.

9. Fiscal. Indica el nombre del agente del Ministerio Público que participó en el juicio oral con jurado.

10. Abogado. Se trata del nombre del abogado que participó en el juicio. El abogado de oficio designado por los jueces no siempre fue el que participó en el juicio, motivo por el que se decidió indicar solo el abogado participante.

11. Jurados. Detalla el nombre de los 11 jurados que participaron en el juicio oral. Este dato se extrajo principalmente de dos autos: el acta del juicio oral realizada por los escribanos y la lista de sorteo de jurados. Es un dato para referenciar los ciudadanos participantes.

12. Veredicto. Se trata del dato relativo al veredicto sancionado por el jurado durante la sesión de juicio público. Éste se concretaba en el cuestionario realizado por los jueces a los jurados que contenía una serie de preguntas sobre la comisión del hecho 
punitivo y los atenuantes y agravantes del mismo. Era imprescindible una pregunta inicial sobre la comisión del hecho por parte del incriminado, y no había límite sobre el número de preguntas formuladas. Por su parte, los jurados debían responder con las expresiones "sí" o "no" a cada una de las preguntas. El tiempo de resolución debía llevarse a cabo con prontitud procurando evitar el llamado a una nueva sesión. Debido a la importancia atribuida a este dato dentro de la investigación (por su énfasis sobre la participación ciudadana) se procuró transcribir al menos las dos preguntas iniciales realizadas por los jueces. ${ }^{31}$

13. Sentencia. Expone la sentencia penal proferida por el juez a partir del veredicto emitido por el jurado. Para su elaboración el juez enfatizaba el resultado del veredicto y señalaba los artículos penales que fundaban la condena o la absolución. En el catálogo se procuró indicar únicamente los artículos penales indicados por los jueces y el término de la condena o la absolución.

14. Revisión de Corte. Es el auto realizado por el Tribunal Superior del Distrito Federal de México que era la segunda instancia para los delitos penales comunes. En el registro de catálogo se indicó exclusivamente la confirmación o la modificación.

15. Descripción. Resumen basado en los datos de los pleiteantes, el móvil del delito y la sanción. Se indican aspectos del funcionamiento del jurado, como la reiteración de sorteos, y se subrayan elementos notables del delito.

16. Estado material. Se estableció para referir cortes, hongos, manchas u otros daños materiales que presenten los folios de los expedientes.

17. Folios. El número de folio se implantó para dar cuenta del número total de folios de los expedientes criminales.

Sobre la regulación del veredicto véase el segundo apartado. 
Tabla 2. Ejemplo de ejercicio de catálogo de expedientes criminales con Jurado del Fondo Tribunal Superior de Distrito Federal de México D. F. 1869-1883

\begin{tabular}{|c|c|c|}
\hline \multicolumn{2}{|c|}{ Núm. Caja } & C-473 \\
\hline \multicolumn{2}{|c|}{ Núm. Expediente } & 1011 \\
\hline \multicolumn{2}{|c|}{ Cronología } & $19 / 12 / 1869-01 / 10 / 1870$ \\
\hline \multicolumn{2}{|c|}{ Delito } & homicidio \\
\hline \multicolumn{2}{|c|}{ Lugar } & Ciudad de México \\
\hline \multicolumn{2}{|c|}{ Demandante } & Francisco López \\
\hline \multicolumn{2}{|c|}{ Demandado } & Magdaleno Barajas \\
\hline \multirow{3}{*}{$\begin{array}{c}\text { Burocracia } \\
\text { Judicial }\end{array}$} & Juez & Lic. José María Castellanos \\
\hline & Fiscal & Lic. Camalizo \\
\hline & Abogado & Manuel Guillermo Prieto \\
\hline \multirow[b]{2}{*}{$\begin{array}{c}\text { Participación } \\
\text { ciudadana }\end{array}$} & Jurados & $\begin{array}{l}\text { Juan Rondero, Luis Veraza, Pedro R. del Valle, Paz Reyes, } \\
\text { José María Rodríguez, Andrés del Río, Benito Quintana, } \\
\text { [Eurigio] Palacios, Manuel Santa María, M. Onofre Pare- } \\
\text { des, Carlos Benites y Piña. }\end{array}$ \\
\hline & Veredicto & $\begin{array}{c}1 \text { ¿Es culpable Magdaleno barajas del homicidio de Francis- } \\
\text { co López? Sí, por ocho votos. } \\
2 \text { ¿Esta herida fue inferida en riña o pelea? Sí, por nueve } \\
\text { votos. } \\
3{ }_{\dot{C} \text { Hubo grave provocación por parte de López, que produ- }} \\
\text { jera aumento en el ánimo de Barajas? No, por ocho votos. } \\
4 \text { ¿Se hallaba ebrio Magdaleno Barajas? Sí, por siete votos. } \\
5 \text { ¿Esta ebriedad era completa? No, por diez votos. }\end{array}$ \\
\hline \multirow{2}{*}{$\begin{array}{c}\text { Codificación } \\
\text { y arbitrio }\end{array}$} & Sentencia & $\begin{array}{c}\text { Comprendido este caso en los Artículos } 30 \text { y } 32 \text { de la Ley } \\
\text { de } 5 \text { de enero de } 1857 \text {. Condeno a Magdaleno Barajas a } \\
\text { tres años de prisión en la Cárcel Nacional. Sin responsabili- } \\
\text { dad civil por no haber reclamante. }\end{array}$ \\
\hline & $\begin{array}{l}\text { Revisión } \\
\text { de Corte }\end{array}$ & $\begin{array}{l}\text { Se revoca aumentando la pena a cinco años con base en } \\
\text { los Artículos } 30 \text { y fracción primera del } 32 \text { de la Ley del } \\
5 / 01 / 1857\end{array}$ \\
\hline \multicolumn{2}{|c|}{ Descripción } & $\begin{array}{l}\text { Magdaleno Barajas asesina a Francisco López por una dispu- } \\
\text { ta motivada por un alegato menor y en estado de embria- } \\
\text { guez. El occiso muere en el hospital tras indicar los móviles. } \\
\text { Pudo haber sido condenado el agresor a pena de muerte. }\end{array}$ \\
\hline \multicolumn{2}{|c|}{ Est. de Docu. } & Buen estado, Juicio completo. \\
\hline \multicolumn{2}{|c|}{$\mathbf{N}^{\circ}$ Folios } & 56 \\
\hline
\end{tabular}




\begin{tabular}{|c|c|c|}
\hline \multicolumn{2}{|c|}{ Núm. Caja } & c-473 \\
\hline \multicolumn{2}{|c|}{ Núm. Expediente } & 488 \\
\hline \multicolumn{2}{|c|}{ Cronología } & $28 / 6 / 1869-20 / 01 / 1870$ \\
\hline \multicolumn{2}{|c|}{ Delito } & Estupro \\
\hline \multicolumn{2}{|c|}{ Lugar } & México \\
\hline \multicolumn{2}{|c|}{ Demandante } & Casimiro Hernández \\
\hline \multicolumn{2}{|c|}{ Demandado } & Cipriana Calderón \\
\hline \multirow{3}{*}{$\begin{array}{c}\text { Burocracia } \\
\text { Judicial }\end{array}$} & Juez & Lic. José María Castellanos \\
\hline & Fiscal & Covarrubias \\
\hline & Abogado & Viqueras \\
\hline \multirow[b]{2}{*}{$\begin{array}{l}\text { Participación } \\
\text { ciudadana }\end{array}$} & Jurados & $\begin{array}{l}\text { Manuel Marquecho, José María Malo, Vicente Macías, } \\
\text { Luis Méndez, Vicente Montes de Oca, Luis Landa, Félix } \\
\text { Marques, Ángel Morales, Antonio Lara, Pedro Mendoza, } \\
\text { Leonardo Pietra Santa. }\end{array}$ \\
\hline & Veredicto & $\begin{array}{c}1 \text { ¿Es culpable Casimiro Hernández del estupro inmaturo } \\
\text { perpetrado en la persona de Cipriana Calderón } \\
\text { Sí por ocho votos. } \\
2 \text { ¿Es culpable de fuerza en la misma Cipriana Calderon? } \\
\text { No por ocho votos. }\end{array}$ \\
\hline \multirow{2}{*}{$\begin{array}{c}\text { Codificación } \\
\text { y arbitrio }\end{array}$} & Sentencia & $\begin{array}{l}\text { Novísimo tomo } 1^{\circ} \text {. Prontuario de delitos, palabra } \\
\text { estupro, Escriche, diccionario de legislación. Partida } 7 \text {. } \\
\text { Fallo la pena de dos años de presidio. y pago de } \\
15 \text { pesos de dote á Cipriana Calderón. }\end{array}$ \\
\hline & $\begin{array}{l}\text { Revisión } \\
\text { de Corte }\end{array}$ & Confirmada \\
\hline \multicolumn{2}{|c|}{ Descripción } & $\begin{array}{c}\text { Se prueba la culpabilidad del guarda faroles. Hay testigos } \\
\text { para la detención, y rastros de sangre en pantalón. El fiscal } \\
\text { pide la condenación. El delito fue causado con fuerza y arma. }\end{array}$ \\
\hline \multicolumn{2}{|c|}{ Est. de Docu. } & Buen estado. No presenta caratula. Expediente Completo. \\
\hline \multicolumn{2}{|c|}{$\mathbf{N}^{\mathbf{0}}$ Folios } & 55 \\
\hline
\end{tabular}




\begin{tabular}{|c|c|c|}
\hline \multicolumn{2}{|c|}{ Núm. Caja } & C-4921870 \\
\hline \multicolumn{2}{|c|}{ Núm. Expediente } & [no] \\
\hline \multicolumn{2}{|c|}{ Cronología } & $13 / 09 / 1870-15 / 11 / 1870$ \\
\hline \multicolumn{2}{|c|}{ Delito } & Se encabeza como plagio se juzga como hurto. \\
\hline \multicolumn{2}{|c|}{ Lugar } & Ciudad de México \\
\hline \multicolumn{2}{|c|}{ Demandante } & Manuel Garcés \\
\hline \multicolumn{2}{|c|}{ Demandado } & Dámaso Almazán \\
\hline \multirow{3}{*}{$\begin{array}{l}\text { Burocracia } \\
\text { Judicial }\end{array}$} & Juez & Lic. Gaxiola \\
\hline & Fiscal & Martín García Esqueta \\
\hline & Abogado & Manuel Prieto \\
\hline \multirow[b]{2}{*}{$\begin{array}{l}\text { Participación } \\
\text { ciudadana }\end{array}$} & Jurados & $\begin{array}{c}\text { Miguel María Azcarate, Francisco Abadiano, Manuel } \\
\text { Cevallos, Miguel Corral, José María Aguilar, Julio } \\
\text { [Arancioria] José María Cuevas, Alberto Carrera, Miguel } \\
\text { Cordero, Alberto Aguado. }\end{array}$ \\
\hline & Veredicto & $\begin{array}{c}1 \text { ¿Es culpable Dámaso Almazán del delito } \\
\text { de robo que se cometió en unos objetos del uso personal de } \\
\text { Víctor Manuel Garcés? Sí por diez votos. } \\
2 \text { ¿Hubo violencia? Sí por ocho votos. } \\
3^{\circ} \text { ¿La violencia se ejerció en una persona indefensa? } \\
\text { Sí por unanimidad }\end{array}$ \\
\hline \multirow{2}{*}{$\begin{array}{l}\text { Codificación } \\
\text { y arbitrio }\end{array}$} & Sentencia & $\begin{array}{l}\text { Comprendido este caso en el Art. } 43 \text { de la ley } \\
\text { (05-01-1857), se condena a Dámaso Almazán por el delito } \\
\text { de robo a la pena de cinco años de presidio con descuento } \\
\text { de la prisión sufrida. }\end{array}$ \\
\hline & $\begin{array}{l}\text { Revisión } \\
\text { de Corte }\end{array}$ & $\begin{array}{l}\text { Se revoca con base en el Art. 43. Indicado en la sentencia. } \\
\text { Se condena a cuatro años de presidio. }\end{array}$ \\
\hline \multicolumn{2}{|c|}{ Descripción } & $\begin{array}{l}\text { El niño Víctor Manuel Garcés sostuvo que el procesado lo } \\
\text { saco cargado del patio del colegio contra su voluntad. El } \\
\text { reconocimiento se dio entre varios reos, logrando identificar } \\
\text { a Almazán por su rostro. Almazán fue capturado infraganti } \\
\text { vendiendo las ropas en el baratillo. }\end{array}$ \\
\hline \multicolumn{2}{|c|}{ Est. de Docu. } & Buen estado. Expediente completo. \\
\hline \multicolumn{2}{|c|}{$\mathbf{N}^{\circ}$ Folios } & 49 \\
\hline
\end{tabular}




\begin{tabular}{|c|c|c|}
\hline \multicolumn{2}{|c|}{ Núm. Caja } & c-503 \\
\hline \multicolumn{2}{|c|}{ Núm. Expediente } & 294 \\
\hline \multicolumn{2}{|c|}{ Gronología } & $10 / 03 / 1870-14 / 06 / 1870$ \\
\hline \multicolumn{2}{|c|}{ Delito } & Heridas que derivan en homicidio \\
\hline \multicolumn{2}{|c|}{ Lugar } & México \\
\hline \multicolumn{2}{|c|}{ Demandante } & Isabel Díaz \\
\hline \multicolumn{2}{|c|}{ Demandado } & Jesús Espinosa \\
\hline \multirow{3}{*}{$\begin{array}{l}\text { Burocracia } \\
\text { Judicial }\end{array}$} & Juez & Lic. José María Castellanos \\
\hline & Fiscal & Pedro Covarrubias \\
\hline & \begin{tabular}{|l|} 
Abogado \\
\end{tabular} & Viviano Beltrán \\
\hline \multirow[b]{2}{*}{$\begin{array}{l}\text { Participación } \\
\text { ciudadana }\end{array}$} & Jurados & $\begin{array}{c}\text { Jesús Reyna, Vicente Santillán, Luis Isidro, Mariano } \\
\text { Rangel, José de la Torre, José Villanueva, Juan Salazar, } \\
\text { Eduardo Zalazar, Aureliano Rivera, Rafael Ríos hijo, } \\
\text { Anastasio Sirón. }\end{array}$ \\
\hline & Veredicto & $\begin{array}{l}1 \text { ¿Es culpable Jesús Espinoza de la herida que se infirió } \\
\text { a Isabel Díaz? Sí por unanimidad. } 2 \text { ¿Infirió esta herida a } \\
\text { Díaz tratando de herir a otra persona? Sí por unanimidad. } \\
3 \text { ¿Se causó la herida en riña o pelea? Sí por unanimidad. } \\
4 \text { ¿Estaba ebrio Jesús Espinoza? Sí por unanimidad. } 5 \text { ¿Esta } \\
\text { ebriedad era completa? Sí por unanimidad. } 6 \text { ¿tenía la } \\
\text { intención de causar un mal menor? Sí por unanimidad. }\end{array}$ \\
\hline \multirow[b]{2}{*}{$\begin{array}{c}\text { Codificación } \\
\text { y arbitrio }\end{array}$} & Sentencia & $\begin{array}{l}\text { Art } 14 \text { fra. } 2 \text { y } 35 \text { de } 05 / 01 / 1857 \text {. Fracción } 3 \text { del Art. } 32 \text { y } \\
55 \text { del Art. } 6 \text {. Absolución. }\end{array}$ \\
\hline & $\begin{array}{l}\text { Revisión } \\
\text { de Corte }\end{array}$ & $\begin{array}{l}\text { Veredicto nulo. Corrección de corte: Se ha declarado en } \\
\text { la pregunta } 5^{\circ} \text { que Espinosa estaba ebrio. Cuando se dice } \\
\text { en la } 2^{\circ} \text { que hirió a Díaz Tratando de herir a otra persona. } \\
\text { "El juez de derecho debe apreciar las declaraciones del jura- } \\
\text { do en su sentido literal y no buscar el que pudieran haberle } \\
\text { dado atentas las constancias del proceso, pues de no ser así } \\
\text { se minaría por su base la institución del Jurado." } \\
\end{array}$ \\
\hline \multicolumn{2}{|c|}{ Descripción } & $\begin{array}{l}\text { El acusado es culpable de inferir heridas a una mujer } \\
\text { cuando intentaba golpear a otras mujeres con las que reñía. } \\
\text { Se declara el veredicto como contradictorio, y se resalta la } \\
\text { soberanía del jurado en la determinación del hecho. }\end{array}$ \\
\hline \multicolumn{2}{|c|}{ Est. de Docu. } & $\begin{array}{l}\text { Completo el primer juicio en que se declaró inocente. } \\
\text { Falta la apelación. }\end{array}$ \\
\hline \multicolumn{2}{|c|}{$\mathbf{N}^{\circ}$ Folios } & 70 \\
\hline
\end{tabular}




\begin{tabular}{|c|c|c|}
\hline \multicolumn{2}{|c|}{ Núm. Caja } & C-599 \\
\hline \multicolumn{2}{|c|}{ Núm. Expediente } & 10364 \\
\hline \multicolumn{2}{|c|}{ Cronología } & $01 / 04 / 1876-11 / 09 / 1876$ \\
\hline \multicolumn{2}{|c|}{ Delito } & Conato de fuga y homicidio \\
\hline \multicolumn{2}{|c|}{ Lugar } & Ciudad de México \\
\hline \multicolumn{2}{|c|}{ Demandante } & Francisco Sánchez (occiso) \\
\hline \multicolumn{2}{|c|}{ Demandado } & Domingo Hernández \\
\hline \multirow{3}{*}{$\begin{array}{l}\text { Burocracia } \\
\text { Judicial }\end{array}$} & Juez & Joaquín M. Escoto \\
\hline & Fiscal & Faustino Michel \\
\hline & Abogado & Francisco F. Gordillo \\
\hline \multirow[b]{2}{*}{$\begin{array}{l}\text { Participación } \\
\text { ciudadana }\end{array}$} & Jurados & $\begin{array}{l}\text { José Fuentes, Eutimio Gómez, Martín Gaytán, Felipe } \\
\text { Escalante, Enrique León, Juan Gutiérrez, Francisco } \\
\text { Moreno, José Ibarra, Lázaro Murillo, Mariano Lara, } \\
\text { Rafael González. }\end{array}$ \\
\hline & Veredicto & $\begin{array}{l}1_{\mathrm{C} E s} \text { culpable Domingo Hernández del homicidio perpetra- } \\
\text { do en la persona de Francisco Sánchez la madrugada del } \\
\text { día } 1^{\circ} \text { de abril último? No por diez votos contra uno }\end{array}$ \\
\hline \multirow[b]{2}{*}{$\begin{array}{c}\text { Codificación } \\
\text { y arbitrio }\end{array}$} & Sentencia & $\begin{array}{l}\text { Absuelto. Con fundamento al Art. } 49 \text { de la Ley de } 15 \text { de } \\
\text { junio de } 1869 \text { se mandó poner en libertad al acusado. }\end{array}$ \\
\hline & $\begin{array}{l}\text { Revisión } \\
\text { de Corte }\end{array}$ & Confirmada \\
\hline \multicolumn{2}{|c|}{ Descripción } & $\begin{array}{l}\text { Se desarrolla un tumulto organizado por parte de algunos } \\
\text { reos de la cárcel de Belem. Dos hombres asesinan al soldado } \\
\text { Francisco Sánchez. Fueron sorprendidos por Domingo } \\
\text { Hernández otro guardia. }\end{array}$ \\
\hline \multicolumn{2}{|c|}{ Est. de Docu. } & $\begin{array}{c}\text { Buen estado. Juicio completo. Algunas fojas no están adheri- } \\
\text { das al cosido del expediente. }\end{array}$ \\
\hline \multicolumn{2}{|c|}{$\mathbf{N}^{\mathbf{0}}$ Folios } & 58 \\
\hline
\end{tabular}




\section{Conclusiones}

En este artículo se estableció, en su primera parte, que el proceso de institucionalización del jurado en la Ciudad de México para delitos comunes tuvo una discusión reiterada desde el Periodo Independentista y que en la sanción de la primera Ley Orgánica de Jurados para la materia de 1869 incidió la perspectiva que consideraba el jurado como un mecanismo generador de la participación en la justicia. En esta parte, también se profundizó en el aspecto relativo a que la institución contribuyó a la dinamización del gobierno local, tal y como lo indica la abundante documentación sobre los procesos electorales realizados por el cabildo y los expedientes criminales. En este sentido, el análisis realizado reafirmó la tesis sostenida desde la historiografía del derecho y de la justicia sobre el pluralismo jurídico y judicial generado por el jurado en tanto se trató de una justicia administrada por particulares. ${ }^{32}$ Este pluralismo en el ámbito judicial -que no hacía exclusiva la aplicación de la ley- se fundaba en el reconocimiento del jurado como un mecanismo de representación ciudadana en el que se aplicaba la teoría de la soberanía popular.

En segundo lugar, en este artículo pudo determinarse que el principal valor del expediente criminal como fuente histórica sobre el jurado popular radica en la información que aporta sobre la participación en la justicia. Esto lo convierte en una fuente idónea para dar cuenta, cuando son analizados corpus amplios, de las tendencias ciudadanas generadas por la institución en un ámbito dinámico como el del juicio oral. En este sentido, el análisis de los expedientes puede aportar ampliamente al debate actual sobre el sistema penal acusatorio en México, en la medida en que ilustran sobre la aplicación de garantías procesales como el sorteo, la recusación y el nombramiento de abogados, y también sobre el funcionamiento de principios como los de inmediación, concentración, contradicción y veredicto inapelable sobre el hecho.

${ }^{32}$ Speckman, "Los jueces, el honor y la muerte", p. 146. 
Finalmente, en este artículo se ha precisado que los expedientes criminales con jurado del FTSJDF son un género documental con unas características de forma y contenido definidas y que éstos destacan por su notable volumen, variedad y buen estado material. El trabajo realizado en dicho fondo permitió la identificación y digitalización de un corpus de 713 expedientes criminales tramitados entre 1869 y 1883. El estudio del conjunto, por su parte, indicó que predominaron los expedientes por delitos de lesiones, robo y homicidio, y ellos se encuentran en buen estado de conservación. Respecto al trabajo de catalogación, se ha logrado un registro de 282 expedientes y se espera terminar todo el conjunto de 713 para realizar la publicación respectiva. En el futuro se espera también generar proyectos que permitan la identificación y catalogación de los expedientes posteriores a 1883. 


\section{Fuentes}

\section{Archivos}

AGN-México Archivo General de la Nación de México.

FTSJDF Fondo Tribunal Superior de Justicia del Distrito Federal.

\section{Bibliografía}

Arnold, Linda, Catálogo de Documentos Ayuntamiento de la Ciudad de México, Fusticia II. Ciudad de México, Gobierno del Distrito Federal, 2000.

Arroyo Padilla, Antonio, "Los jurados populares en la administración de la justicia en México en el siglo xix”, en Secuencia, núm. 47, 2000, México, Instituto Mora.

Bentham, Jeremy, Tratados de legislación civil y penal (traducida y comentada por Ramón de Salas, bajo la dirección de José René Masson), París, Imprenta Masson e Hijos, 8 vol. 1823.

Clavero, Bartolomé, Happy Constitution. Cultura y lengua constitucionales, Madrid, Trotta, 1997.

Constant, Benjamin, Curso de política constitucional, Madrid, Imprenta de la Compañía, 3 vol. 1821.

Dublán Manuel y Lozano, José María, Legislación mexicana. Colección completa de las disposiciones legislativas expedidas desde la Independencia de la República, Edición oficial, México, Imprenta del Comercio de Dublán y Chávez. (1821-1891), t. x.

Escriche, Joaquín, Diccionario Razonado de Legislación y Jurisprudencia, Tercera Edición Corregida y Aumentada, Madrid, 1847. 
Examen histórico-crítico de la institución del Furado, Imprenta del Colegio Nacional de Sordo-Mudos, Madrid, 1844.

Filangieri, Gaetano, Ciencia de la legislación, t. Iv, París, 1836.

Flores Flores, Graciela, "Sobre la fundamentación de las sentencias y el arbitrio judicial: un recuento de la larga marcha hacia la codificación en la Ciudad de México, siglo xix", en Passagens Revista Internacional de História Política e cultura jurídica Río de Faneiro, vol. 8, núm. 2, mayo-agosto de 2016, pp. 206-232.

Garriga, Carlos y Lorente Marta, Cádiz, 1812. La constitución jurisdiccional, Madrid, Centro de Estudios Políticos y Constitucionales, 2007.

, Historia y Constitución, Trayectos del constitucionalismo hispano, cide/El Colegio de México, 2010.

González Oropeza, "El juicio por Jurado en las constituciones de México", en Cuestiones Constitucionales, núm. 2, México, UNAM, 2000.

González Villalobos, Pablo Héctor, "Sistemas penales y reforma procesal penal en México", en Justice in Mexico, Working Paper Series, vol. 14, núm. 3, 2015.

González Florentino, El Juicio por Furados. Breve noticia del origen y progresos del jurado, del modo de practicar la prueba judicial en Inglaterra y los Estados Unidos, comparado con otras naciones; y razones en favor de esta institución. Extracto de los mejores autores que tratan sobre la materia, Buenos Aires, Imprenta y Fundición de Tipos a Vapor, 1869.

Hale, Charles A., El liberalismo mexicano en la época de Mora 1821-1853, México, Siglo xxi, 1977. 
Hernández, Eskándar Gánem, "La reforma procesal penal federal en México" (tesis de doctorado), defendida en Universidad Jaume i de Castellón, España, 2012.

Irurozqui Victoriano, Marta, "El espejismo de la exclusión. Reflexiones conceptuales acerca de la ciudadanía y el sufragio censitario a partir del caso boliviano", en Ayer, núm. 70, 2008.

, La ciudadanía en debate en América Latina. Discusiones historiográficas y una propuesta teórica sobre el valor público de la infracción electoral, Lima, IEP, 2005.

, Sangre de ley (Introducción), Ediciones Polifemo, Madrid, 2011.

Lira González, Andrés, El Amparo colonial y el Fuicio de Amparo Mexicano: antecedentes novohispanos del Fuicio de Amparo, FCE, 1972.

Londoño Tamayo, Andrés Alejandro, "El Jurado popular en Colombia en el juicio criminal ordinario. Participación ciudadana y justicia penal en Medellín (1821-1886)", en Revista de Indias, vol. Lxxvi, núm. 266, pp. 203-232.

, "Juicios de imprenta en Colombia (1821-1851). El jurado popular y el control de los libelos infamatorios", en Anuario Colombiano de Historia Social y de la Cultura, vol. 40, núm. 1, 2013, pp. 75-112.

López, Georgina, La organización para la administración de la justicia ordinaria en el segundo imperio, México, El Colegio de México, 2014 .

Marino, Daniela, Huixquilucan ley y justicia en la modernización del espacio rural mexiquense, 1856-1910, Madrid, Consejo Superior de Investigaciones Científicas, 2016. 
México Distrito Federal, Ley de jurados en materia criminal para el Distrito Federal, México, J. S. Ponce de León, Impresor, 1869.

México Distrito Federal, Código Penal para el Distrito Federal y territorio de la Baja California sobre delitos del fuero común, y para toda la República sobre delitos contra la Federación, Veracruz, Imprenta del progreso, 1873.

Monroy Nasr, Rebeca, "Identidades perdidas: Miss México 1928", en Anales del Instituto de Investigaciones Estéticas, vol. xxxvi, núm. 104, 2014, pp. 127-156.

Mora, José María, "Obras sueltas”, en Revista Política-Crédito público, t. 1, París, Librería de Rosa, 1837.

Ovalle, Favela, "Los antecedentes del jurado popular en México" en Criminalia, núm. 7-9, 1981, pp. 61-94.

Piccato, Pablo, Ciudad de sospechosos. Crimen en la Ciudad de México 1900-1931, México, ciesas, 2010.

Rabasa, Emilio O., "El jurado popular en las constituciones de 1857 y 1917. Antecedentes, secuencia y consecuencias", en Liber ad honorem. Sergio García Ramirez, México, unam, 1998, t. I.

Rojas Sosa, Odette María, "El caso de la fiera humana, 1929. El crimen de la calle de Matamoros, el nuevo Código Penal y la desaparición del Jurado Popular", en Historia y Grafía, núm. 30, 2008, pp. 217-245.

Sodi, Demetrio, El jurado popular en México: estudios sobre el jurado popular, México, Imprenta de la Secretaría de Fomento, 1909.

Speckman Guerra, Elisa, "El Jurado popular para delitos comunes: leyes, ideas y prácticas (distrito federal, 1869-1929)", en Cárdenas, 
Salvador (ed.), Historia de la justicia en México (siglos XIX y XX), México, Suprema Corte de Justicia, 2005.

, Del Tigre de Santa Julia, la princesa italiana $y$ otras historias. Sistema judicial, criminalidad y justicia en la Ciudad de México, INACIPE-Históricas UNAM, 2015.

, "Los jueces, el honor y la muerte. Un análisis de la justicia (Ciudad de México, 1871-1931)", en Historia Mexicana, núm. 4. México, 2006.

Zavala, Lorenzo, Viaje a los Estados Unidos de Norte América, París, Imprenta de Decourchant, 1834.

Zarco, Francisco, Historia del Congreso Constituyente de 1857, México, INEHRM, Gobierno del estado de Puebla, 1987. 